\title{
Achieving High-resolution of Large Specimens Using Aberration-corrected Tomography
}

Reed Yalisove ${ }^{1}$, Suk Hyun Sung ${ }^{1}$, Jonathan Schwartz ${ }^{1}$, Catherine Groschner ${ }^{2}$, Philipp Pelz ${ }^{2}$, Huihuo Zheng $^{3}$, Yi Jiang ${ }^{3}$, Colin Ophus ${ }^{4}$, Mary Scott ${ }^{4}$, Peter Ercius ${ }^{4}$ and Robert Hovden ${ }^{1}$

${ }^{1}$ University of Michigan, Ann Arbor, Michigan, United States, ${ }^{2}$ University of California Berkeley, Berkeley, California, United States, ${ }^{3}$ Argonne National Laboratory, Lemont, Illinois, United States, ${ }^{4}$ Lawrence Berkeley National Laboratory, Berkeley, California, United States

Aberration-corrected electron microscopy can resolve the smallest atomic bond-lengths in nature [1-3]. However, the high-convergence angles that enable spectacular resolution in 2D can only achieve limited 3D atomic resolution for all but the smallest objects (c.a. $5-10 \mathrm{~nm}$ ). We show aberration-corrected electron tomography can offer new limits to 3D imaging by sampling several focal planes at each specimen tilt. We present a theoretical foundation for aberration-corrected electron tomography by establishing analytic descriptions for resolution, sampling, object size, and dose — with direct analogy to the CrowtherKlug criterion.

Remarkably, aberration-corrected scanning transmission electron tomography can offer dose-efficient 3D reconstruction that measures complete specimen information of unbounded object sizes up to a specified cutoff resolution [4]. With aberration-corrected electron tomography, defocus and specimen tilt are combined (Figure 1a,b). This breaks the long-accepted Crowther limit when the specimen tilt increment is twice the convergence angle or smaller. When the tilt angle matches the convergence angle, 3D resolution is half the 2D resolving power across any object size. For finite object sizes, unprecedented limits to $3 \mathrm{D}$ resolution are achievable. Atomic resolution $(1.5 \AA) 3 \mathrm{D}$ imaging is allowed across extended objects ( $>15 \mathrm{~nm}$ ) using currently available microscopes and modest specimen tilting $\left(3^{\circ}\right)$ and verified here by quantum mechanical scattering simulations.

Here we present large-scale multislice simulations run on high performance computing systems to validate the theoretical limits of aberration-corrected tomography and demonstrate 3D atomic resolution imaging of extended specimens. The simulated object incorporates three FePt nanoparticles, inspired by Yang et al. [5], arranged to occupy a $15 \mathrm{~nm}$ cube in 3D. Images were calculated with a fully quantum-mechanical multiple scattering simulation [6] for each tilt and defocus. The multislice algorithm available in the Prismatic package [7] provides GPU accelerated implementations and parallelization across multiple cores. This simulation consists of over 1300 projection images at 13 defoci (figure 1a) and 105 tilt increments (figure 1b) that requires a total of $\sim 500$ million wavefunctions to propagate through the $15 \mathrm{~nm}$ specimen. The simulated data was used to tomographically reconstruct all three nanoparticles positioned at varying z-heights within the simulation at atomic resolution (Fig. 1c).

In aberration-corrected tomography each through-focal stack is mapped to Fourier (k-) space to fill information within a 3D contrast transfer function (CTF) [4]. The object is reconstructed from a simple inverse Fourier transform. Figure 1c shows an atomic resolution aberration-corrected tomography reconstruction from simulated data across a $15 \mathrm{~nm}$ field of view in three dimensions. Here, aberrationcorrected tomography allows a reconstruction nearly 5 times larger than the Crowther criterion [8] prediction for traditional tomography.

Surprisingly, aberration-corrected tomography is not necessarily dose intensive. Despite the large amount of image data required by aberration-corrected electron tomography, the dose can be chosen to mitigate 
total specimen exposure. Extending the dose fractionation arguments presented by Hoppe [9] and Saxton [10], we show aberration-corrected electron tomography allows tunable dose allocation across any number of tilts and focal planes when information is oversampled. Multislice simulation demonstrates the dose fractionation theorem for through-focal imaging in Figure 2. By adding Poisson noise, we simulate several low dose images spanning a single depth-of-focus $(\sim 2.8 \mathrm{~nm})$. The average of these low-dose images is compared to a single high-dose image (Fig. 2e) with equivalent total dose (Fig. 2c,d). Each carry identical information. As expected, the SNR is equivalent for several averaged images across defoci at lower dose or a single high-dose image. We extend this to the full range of defocus. When the through-focal stack is evenly oversampled along defocus, the quality of the reconstruction is dependent only on the total dose, not the distribution of dose. Unfortunately, we anticipate aberration corrected tomography follows traditional dose requirements where $3 \mathrm{D}$ resolution scales inversely with $\operatorname{dose}^{\wedge} 1 / 4[10,11]$ and atomic resolution requires substantial beam exposure.

With aberration-corrected electron tomography, we can proceed to higher resolution across larger fieldsof-view to know the structure of extended specimens in all three dimensions [12-14].


Figure 1. a) Through-focal image stack highlighting limited depth of focus in STEM imaging for a $200 \mathrm{keV}$ probe with $30 \mathrm{mrad}$ convergence angle. No single image captures all nanoparticles in focus simultaneously. b) Our simulation collects a through-focal image stack at each tilt angle. c) Aberrationcorrected electron tomography reconstruction with atomic resolution. 




Figure 2. Dose fractionation for through-focal acquisition a) Adjacent defocused images in through-focal image stack can summed without any loss of information as long as defocus sampling requirement is met. b) Defocus sampling requirement is set by the depth-of-focus. Multislice simulation with Poisson noise shows the equivalence of SNR in c,d) sum of low dose defocused images and e) a single high dose image.

\section{References}

[1] P. E. Batson, N. Delby, and O. L. Krivanek, Nature 418 (2002) p. 617.

[2] P. D. Nellist, et al. Science 305 (2004) p. 1741.

[3] D. A. Muller, et al. Science 319 (2008) p. 1073.

[4] R. Yalisove, S. H. Sung, R. Hovden, Microsc. Microanal. 25(Suppl 2) (2019) p. 1810

[5] Y. Yang et alNature 542 (2017) p. 75-79.

[6] J.M. Cowley, A.F. Moodie. Acta Cryst. 10 (1957) p. 609-619.

[7] A. Pryor, C. Ophus, J. Miao Adv. Struc. Chem. Imag. 3 (2017) p.15.

[8] R. Crowther, D. DeRosier, A. Klug, Proc. R. Soc. A 317 (1970) p. 319-340.

[9] R. Hegerl, W. Hoppe, Zeitschrift für Naturforschung A 31(12) (1976) p. 1717-1721.

[10] B. Saxberg, W. Saxton, Ultramicroscopy 6 (1981) p. 85-90.

[11] B. F. McEwen, et al., Journal of Structural Biology 138 (2002) p. 47.

[12] This research used resources of the Oak Ridge Leadership Computing Facility, which is a DOE Office of Science User Facility supported under Contract DE-AC05-00OR22725.

[13] Work at the Molecular Foundry was supported by the Office of Science, Office of Basic Energy Sciences, of the U.S. Department of Energy under Contract No. DE-AC02-05CH11231.

[14] This research used resources of the Argonne Leadership Computing Facility, which is a DOE Office of Science User Facility supported under Contract DE-AC02-06CH11357, and resources of the Oak Ridge Leadership Computing Facility, which is a DOE Office of Science User Facility supported under Contract DE-AC05-00OR22725. 